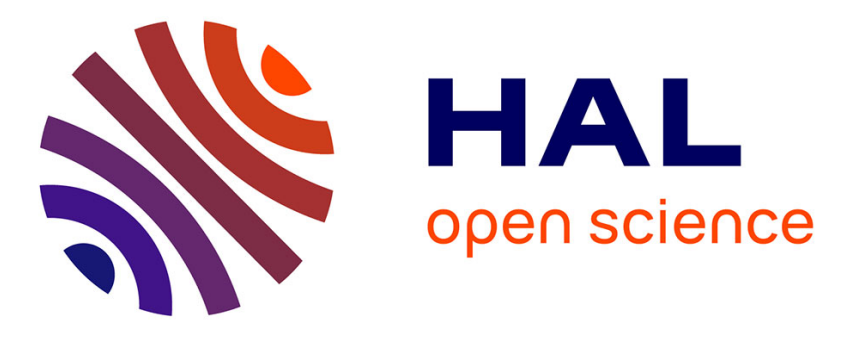

\title{
Sustainable ICT4D in Africa: Where Do We Go From Here?
}

Tegawendé F. Bissyandé, Daouda Ahmat, Jonathan Ouoba, Gertjan van

Stam, Jacques Klein, Yves Le Traon

\section{- To cite this version:}

Tegawendé F. Bissyandé, Daouda Ahmat, Jonathan Ouoba, Gertjan van Stam, Jacques Klein, et al.. Sustainable ICT4D in Africa: Where Do We Go From Here?. Fifth International IEEE EAI Conference on e-infrastructure and e-Services for Developing Countries (AFRICOMM 2013), Nov 2013, Blantyre, Malawi. pp.1-10. hal-00994230

\section{HAL Id: hal-00994230 \\ https://hal.science/hal-00994230}

Submitted on 21 May 2014

HAL is a multi-disciplinary open access archive for the deposit and dissemination of scientific research documents, whether they are published or not. The documents may come from teaching and research institutions in France or abroad, or from public or private research centers.
L'archive ouverte pluridisciplinaire HAL, est destinée au dépôt et à la diffusion de documents scientifiques de niveau recherche, publiés ou non, émanant des établissements d'enseignement et de recherche français ou étrangers, des laboratoires publics ou privés. 


\title{
Sustainable ICT4D in Africa: Where Do We Go From Here?
}

\author{
Tegawendé F. Bissyandé ${ }^{1,2}$, Daouda Ahmat $^{3}$, Jonathan Ouoba ${ }^{3}$ \\ Gertjan van Stam ${ }^{4}$, Jacques Klein ${ }^{1}$ and Yves Le Traon ${ }^{1}$ \\ 1 SnT, University of Luxembourg, Luxembourg \\ \{tegawende.bissyande, jacques.klein, yves.letraon\}@uni.lu \\ 2 FasoLabs, Burkina Faso \\ 3 LaBRI - CNRS, University of Bordeaux, France \\ \{adaouda, jonathan.ouoba\}@labri.fr \\ 4 SIRDC, Zimbabwe \\ gertjan.vanstam@machaworks . org
}

\begin{abstract}
In recent years many researchers in Africa and beyond have devoted considerable resources investigating ways to harness the potential of ICT for improving users' livelihood in developing areas. Topics and domains of interest appear to be broad with recurring themes and solutions. Unfortunately there are no clear research roadmaps on what is urgent and of the state of the art solutions. In this position paper for the AFRICOMM series of conference, we propose to investigate some priorities for ICT4D in Africa. We believe that our work could motivate researchers and create a synergy around a few important challenges of ICT4D in Africa.
\end{abstract}

Key words: ICT4D, Africa, development, research topics and priorities

\section{Introduction}

Information and Communication Technologies have shaped todays' world, and keep drawing the future by enforcing globalization and its associated challenges and opportunities for trade, cultural exchange, and innovation. In developing areas, the frontrunner initiatives to move millions of people out of poverty are recurrently said to be based on ICT. ICT for development (ICT4D) is thus the latest paradigm that occupies researchers, not only in computer sciences, but also in humanities. Unfortunately, except a few reports on experiments, there are very few studies that unequivocally show the success or ICT4D research to improve users livelihood in Africa. Furthermore, and what is more important, there are even fewer empirical studies that suggest critical fields where ICT4D would benefit the populations.

Conferences on ICT4D are now legion. At AFRICOMM, every year, researchers from Africa and beyond are proposing various techniques and approaches for ICT4D. However, as readers of papers from previous sessions of this conference, we always wonder to whom the authors make suggestions of such 
ideas. Indeed, often, the techniques appear to be unrealistic given the available resources and the cultural model in developing countries as well as their priorities. We thus believe that there is a need to advocate for a sustainable ICT4D research. We hope that after a solid discussion on our contributions at the conference, together with all participant researchers we will pave a new roadmap to serve African populations with ideas and solutions (1) that they care about, (2) that could really help them, and (3) that constitute a step towards overcoming the challenges of the digital divide. This position paper is also aimed at rallying young (and not so young) researchers across Africa who wish to partake in ICT4D research. Our contributions in this paper are:

1. we succinctly detail the motivation for this paper, establishing the need for questioning ICT4D research for Africa.

2. we discuss what we believe to be critical avenues of research for Africa, categorizing these topics into short, medium and long term impact-oriented research.

3. finally, we suggest research goals, by distinguishing between urban areas and rural areas in developing regions to show that unlike in developed countries, the ICT4D priorities in such areas are very much different in Africa.

The remainder of this paper is organized as follows. Section 2 provides an indepth discussion of our motivation. Section 3 details some research avenues with their degree of priority for Africa. Section 4 discusses research topics following the context in Africa. Section 5 provides an illustrated discussion on our work and presents a proposition to implement our recommendations. We conclude in Section 6.

\section{Motivation}

The intentional use of communication to foster development is not new. As Heeks has recently described, in the mid-1950s there was already initiative for broadcasting development communication, computing/data processing for back office applications in large organization both of government and private sector in developing countries [1]. Nowadays, mobile phone has shaped its way through to become the archetypal application for ICT4D because of its affordability and because it allows to focus on the poor as producers and innovators of ICT. With the proliferation of data and bandwidth, Hilbert has suggested to now turn our eyes towards Big Data as a new paradigm for ICT4D [2].

There seems therefore to be a consensus on how ICT4D is evolving as a notion. However, in practice this evolution is not clearly witnessed in the improvement of poor people's lives. Furthermore, we note three important concerns with ICT4D research in and for Africa.

Absence of agenda. No agenda has been set on what should be done by the next decade or so to achieve any fixed goal. Even policy makers set vague objectives and then tag 'ICT' as a solution to their goals. In such a context, researchers 
cannot be expected to succeed in unclear endeavors. An agenda would also allow to regularly check the progress, continuously improve the means dedicated, and reassess one's ambitions. The UN ICT Task force attempts to implement such an agenda since 2005 [8]. However, it appears that they set various global and vague goals that mainly concern the government bodies and NGOs that are funded by the UN agency.

Little knowledge on needs and requirements. Second, we note that to the best of our knowledge, there are very few empirical studies that characterize the actual needs of developing countries. There are even fewer studies that discuss the requirements for ICT solutions to be proposed. Such studies however to be prerequisites in other no more important fields of research []. As we discussed in previous work, the particularities of the african context should drive the design of ICT4D solutions [6].

Few return on experiences. What have researchers achieved so far to improve poor people's livelihood? Where have we failed and why? These questions persist for African developing countries because of a serious lack of documentation on failed/successful experiences. Practitioners are often afraid to report on their failures so as to maintain funding, while researchers are not keen to thoroughly validate the real adoption of their solutions, preferring to require that poor people adapt to them, rather than refining their techniques to adapt to the context [].

\section{Critical research avenues}

In this section we discuss research avenues that we see as critical today. We have categorized them so as to ensure that we effectively tackle the issues that we have raised in our detailed motivation (cf. Section 2). First we advocate for empirical studies to shake down all beliefs about the needs of poor people and all wrong hypotheses on the context that could derail the applicability of ICT4D research.

\subsection{Empirical studies}

Is broadband connectivity necessary everywhere in developing areas? Is eLearning even an interesting topic for illiterate people in Africa? Such questions require answers for which we unfortunately do not have definite answers []. ICT4D has this particularity that it employs sophisticated technologies to help people out of poverty. However, there are already a number of researchers, specially in sociology and anthropology, who conduct research on the specificities of developing areas and their inhabitants in order to provide insights that would help NGOs and government bodies to efficiently implement development plans. We believe Information Technology researchers should team up with those researchers to investigate the realities of developing areas and draw out facts that will direct the design of ICT solutions. 
Empirical studies can also help to reduce the approximate (and often wrong) hypotheses that IT researchers make when designing their solutions. As researchers from Africa, we have personal experiences on how people regroup and stay very long close just for greeting each other. Unfortunately, we had a hard time convincing other researchers that the duration of such encounters is sufficient to establish reliable P2P connectivity to transfer sizeable data in an opportunistic scenario [6]. An empirical study on the average duration of contacts among people and their degree of recurrence would significantly help people realize the potential of opportunistic networking in Africa where it would be more suitable to our cultural context than it is in developed countries where it was first invented but remains unleverageable.

\subsection{Return on Experiences}

E-money, e-Learning or tele-medicine are praised everywhere as stornercones of ICT4D applications that are continuously helping the poor people. These praises however are based on very few successes which cannot be generalized. Indeed, most practitioners in many countries are aware that there are many more undocumented failures. When did those failure occur and why? Having answers to those questions will undoubtedly avoid repeated ICT4D design errors, and at the same time significantly reduce the waste of resources.

Return on experiences also participate in archiving the efforts of ICT4D for next generations of researchers. Identifying the landmarks already visited and the paths taken so far by other researchers may help in drawing new (and better) approaches.

\subsection{Research roadmaps}

Use of ICT as a vehicle for sustainable economic development cannot be effective if social conditions are not first improved. Thus, it becomes imperative to first direct ICT initiatives towards such goals. To that respect, we remind the reader that ICTs, in the context of ICT4D, are constituted by tools that facilitate communication, the processing and transmission of information and the sharing of knowledge by electronic means. In this section we suggest a classification of research priorities in terms of short, medium and long term impact needs. As McNamara has stated, experience demonstrates that there is no single solution that will work in all settings [3].

Short Term Impact research: The development of Africa is challenged by deep problems such as pandemic diseases, food and water shortage, wars, and a rapid lost of our valuable heritage. We believe that ICT4D researchers should immediately tackle issues related to these problems.

Health management. Whether it is for reducing maternal death, fighting malaria or responding to HIV spread, ICT is increasingly leveraged. Indeed, ICT initiatives have demonstrated that they can make an impact on improving dissemination public health information, on enabling remote consultation, on facilitating 
collaboration and cooperation among practitioners (e.g., learning best practices, and worker training), on strengthening the capabilities for monitoring threats to public health and administrative systems in health care facilities [3]. There are however no definite ICT answers to support all those needs, and ICT4D research in this area must be further pursued.

Food and Water. Once health is acquired, it cannot be sustained if food and water are lacking or are of bad quality. Around the world, initiatives to use ICT as an enabler of smart management of water and food resources are flourishing. In Africa, the room for improvement is still immense. ICT4D researchers can thus contribute to not only disseminate more efficiently information and advice, but also analyze and forecast food security issues for NGOs and government bodies.

Peace. For some time now, the United Nations Educational, Scientific and Cultural Organization has been advocating the use of ICT to promote peace. Thanks to the revolution in ICTs indeed, there is a real opportunity for "multilogue" to promote mutual understanding and peace. In Africa, there is yet to see ICTbased initiatives for peace building, although governance based on citizens' participation through ICT is increasingly advocated without real-world implementation [7]

Heritage. Finally, an urgent matter that african researchers need to look into is the preservation of the heritage. This includes the landscape, the wild life, as well as the material and immaterial legacy of African ancestors and our elders. In developed countries, national museums are taking full advantage of ICT to spark interest on their cultural heritage. In Africa, animal poaching is alarmingly threatening the Big 5 where ICT can help improve surveillance of national parks [4]. Recent events in Mali where Timbuktu's ancient manuscripts were saved in extremis remind us how fragile heritage in Africa is.

Medium Term Impact research: To support and sustain short term impact research initiatives, ICT4D researchers should also allocate resources to investigate medium term issues.

Enhanced e-Agriculture. First, food security will not be fully ensured if eagriculture is not better implemented. To that end, researchers need to identify opportunities for e-agriculture for illiterate populations, in accordance with the cultural models of developing areas where competition is not necessary the first incentive.

Local e-Government. Improving local participatory governance to promote stability is a noble endeavor. Since ingredients for implementing adapted ICT approaches in developing areas are now in place, ICT4D researchers could invest into proposing ICT-based schemes. These may involve online social communities, information portals, discussion forums, etc. which are implemented with the constraints of the african context, instead of being simple copies of solutions from developed countries. 
Waste management. Another research avenue that needs investigation is the management of waste, specifically electronic waste. Indeed, Africa's mounting electronic waste, or e-waste, is presenting both opportunities and problems that needs to addressed. With millions of poor consumers, Africa is importing many near end-of-life electronic devices, generating a massive waste stream. How can ICT4D researchers help with solutions for analyzing, identifying and recycling these devices before they become a serious environment issue?

new Businesses of Open Source. In previous work, we have discussed how Open Source opportunities were wasted in Africa [5]. Among those opportunities are the possibilities for increasing employment of new graduate students with opensource oriented education that will help them build start ups. Indeed, ICT4D should not only focus on fixing what is broken, it should also participate in building what has never existed.

Long-Term Impact Research: ICT4D should not be short-sighted. Nor should it fail to recognize when a field is not ready for the introduction of some new paradigm. We now discuss two important research scenarios that are widely advertised and that should be (re)thought for the long run.

e-Learning. Education is and must remain a priority in Africa. Traditional classrooms may have exhausted different possibilities for welcoming everyone in acceptable conditions. In that regard, e-Learning by using ICT to assist in the exchange of knowledge can be a solution. Unfortunately, success stories told across the web are very few, as the means to implement e-Learning are often costly in a context where even traditional school faces attendance challenges. One could advocate that new learning media would spark the interest of children. Experience in some countries have shown that such an interest is not a lasting one. The problem with E-learning thus lies within its targets. In rural areas, it serves most for advertisement (and perhaps propaganda?), while in urban areas, it can actually overcome the lack of teaching personnel in some domains.

High Connectivity. Building information highways between cities in Africa will not matter if we have not previously imagined the services that will be run with it. There is "build it and they will come" strategy that can work in areas where everything is a priority. High connectivity requires big investments that most developing african developing cannot afford to make right now. However, because of the rapid pace to which Africa is confronted to globalization, there is a need for ICT4D researchers to start devising applications and scenarios for the various connectivity needs in Africa. Thus, increasing WiFi range and using whitespace technologies will make sense if we can fully take advantage of the new bandwidth that will be delivered.

\section{Mind the Context}

In this section we further discuss in details why a research roadmap for ICT4D is necessary in Africa. In developed countries, almost all areas of a single countries, 
i.e. villages and cities, can be treated equally when offering a new ICT service or setting up a new infrastructure, even if the investments will differ. For example, when introducing the recent $4 \mathrm{G}$ services, operators in Europe just planned a progressive extension of the network to cover all areas. In developing countries, however, the situation would be totally, as the priorities between urban and rural areas are very much dissimilar. We exploring in the following some ICT4D priorities in each context.

\subsection{Rural areas - From survival to living}

Rural areas are the most disadvantaged places in developing countries. People still practice subsistence farming to feed their families. However, traditional agriculture can no longer satisfy the needs of family members, and requires more information from outside world to increase productivity. Research on e-agriculture has thus become a priority for african rural areas. Another challenge for such areas is isolation. The lack of practicable roads and the scarce investment from network operators makes this issue persistent, although it is widely accepted that connecting rural areas with the outside world will significantly improve their livelihood, by enhancing health care, school education, farmer training, etc.

In previous work [6], we have shown how rural areas, because of the cultural model respected by inhabitants, can afford to implement ICT4D approaches based on shared technologies. Opportunistic networking is also a favorable ICT4D paradigm for bringing connectivity into such areas. Finally, ICT4D researchers should investigate the use of new free wireless technologies and the reuse/adaptation of existing open source applications.

\subsection{Urban areas - Towards smart cities}

Urban areas in developing countries face different challenges than their rural counterparts. In African cities, infrastructure investments have already been made, and service launches (and ceases) are witnessed every day. Because the youth attempts to identify with developed countries, ICT4D researchers have a wide range of domains where solutions adapted to existing technologies and constraints are needed. We mention here research avenues and ICT paradigms that can be leveraged to incrementally improve user experience of a connected world.

Payment. Across African cities, few inhabitants have bank accounts and credit cards. Network operators have thus seen an opportunity with mobile phones and the prepaid credit system to push for mobile banking. There seems however to be a monopoly of operators to benefit from the system since payment is done through their Integrated Service Digital Network. With the democratization of Near Field Communication (NFC) on cheap smartphones, there is ample opportunity to create new scenarios of payment. 
Transport. African cities are becoming crowded and the way transport systems are working is not simplifying users' life. ICT4D can improve ticketing system, enhance the dissemination of real time information about traffic, bus hours, etc.

Culture. Young Africans in cities are increasingly unaware of the cultural wealth that surrounds them and that tourists come from far away to visit. An avenue of ICT4D research could be to propose an approach for performing a collaborative census of "must-see" tourist places, and to devise plans to spark the interest of local people.

Social Networking. While online social networking is getting adepts among african populations, Internet connectivity is not fully available anytime and anywhere to readily access worldwide sites. There is therefore a new to use alternative communication vehicles such as SMS on mobile phones to create social networks that reflect our realities and capabilities.

\section{Discussion}

To assess the scope of the AFRICOMM conference with regards to our categorization, we consider all 36 papers accepted at the 2012 session of AFRICOMM and based on their titles and abstracts we count how many papers concern one of our research avenues. Table 1 summarizes our findings. It appears that $83 \%$ of the papers are in line with our priorities of ICT4D for Africa. Only $8 \%$ focus on short-term impact research, while only also $8 \%$ provide insights with empirical studies and returns on experience. Almost $50 \%$ of papers deal with problems related to our category of long term impact research. These findings reveal that there is a need to create a synergy of research to increase the value of ICT4D papers at AFRICOMM, by introducing an equilibrium among the various topics of research that can benefit african populations.

We propose to this end, to have a theme of research every year at AFRICOMM that will allow to focus each time on a specific topic that needs attention. We could also setup a special session (or a workshop) where all ICT4D researchers could join resources and ideas to investigate a specific challenge in Africa.

\section{Conclusion}

The momentum of ICT4D research has been increasing in recent years. Research conferences across the world are publishing proceedings with numerous papers proposing every day new solutions and approaches for leveraging ICT in the development of African countries. However, we note that researchers' efforts are dispersed and no clear agenda is set for delivering solutions. Furthermore, research topics are not necessarily selected based on the real needs on the ground of developing countries. 


\begin{tabular}{lll}
\hline Empirical studies & \# of papers \\
\hline Return on experiences & 3 \\
\hline \multirow{2}{*}{ Short term Impact } & Health management & 3 \\
\cline { 2 - 3 } & Food \& water & 0 \\
\cline { 2 - 3 } & Peace & 0 \\
\cline { 2 - 3 } Medium term Impact & Heritage & 0 \\
\cline { 2 - 3 } & e-Governance & 1 \\
\cline { 2 - 3 } & Waste management & 1 \\
\cline { 2 - 3 } & Open Source & 3 \\
\hline Long term Impact & e-Learning & 1 \\
\cline { 2 - 3 } & High Connectivity & 17 \\
\hline \hline Rural areas & & 3 \\
\hline Urban areas & & 17 \\
\hline
\end{tabular}

Table 1. Categories of papers presented at AFRICOMM'2012

In this paper, we have discussed critical research avenues and recommended the establishment of a research theme for each edition of AFRICOMM in order to create a synergy around some challenges of ICT4D in Africa.

\section{References}

1. R. Heeks. Ict4d 2.0: The next phase of applying ict for international development. Computer, 41(6):26-33, 2008.

2. M. Hilbert. Big data for development: From information- to knowledge societies. Available at SSRN: http://ssrn.com/abstract=2205145, January 2013.

3. kerry McNamara. Improving health, connecting people: The role of icts in the health sector of developing countries. A framework paper, October 2007. 
4. M. O. Mendez, T. F. Bissyandé, K. Somasundar, acques Klein, H. Voos, and Y. L. Traon. Towards Automating Surveillance of National Parks: The Next Killer App of ICT4D with UAVs? Submitted to AFRICOMM'13, 2013.

5. H. Ouattara/sanon, J. Ouoba, and T. F. Bissyandé. Open Source in Africa: An Opportunity Wasted? -Why and How FLOSS Should Make Sense for Africa-. In 4th International IEEE EAI Conference on e-Infrastructure and e-Services for Developing Countries, pages 11-14, Yaoundé, Cameroun, Nov. 2012. student paper.

6. J. Ouoba and T. F. Bissyandé. Leveraging the Cultural Model for Opportunistic Networking in sub-Saharan Africa. In 4th International IEEE EAI Conference on e-Infrastructure and e-Services for Developing Countries, AFRICOMM, pages 1-10, Yaoundé, Cameroun, 2012.

7. I. Saleh. The impact of ict on peace, security \& governace in africa. AoC Media Literacy Clearing House.

8. UN ICT Task Force. Tools for development: Using information and communications technology to achieve the millennium development goals. Working Paper, 2005. 